\title{
Overexpression of FOXM1 is associated with EMT and is a predictor of poor prognosis in non-small cell lung cancer
}

\author{
FEI-FEI KONG ${ }^{1 *}$, ZENG-QIANG QU $^{2 *}$, HAI-HUA YUAN ${ }^{1}$, JIONG-YI WANG $^{1}$, MEI ZHAO $^{1}$, YUE-HUI GUO ${ }^{1}$, \\ JING SHI $^{1}$, XIAO-DI GONG ${ }^{1}$, YOU-LONG ZHU ${ }^{3}$, FENG LIU ${ }^{1}$, WEN-YING ZHANG ${ }^{1}$ and BIN JIANG ${ }^{1}$ \\ ${ }^{1}$ Department of Oncology, No. 3 People's Hospital Affiliated to Shanghai Jiaotong University School of Medicine (SJTU-SM), \\ Shanghai 201900; ${ }^{2}$ Department of Interventional Radiology, Eastern Hepatobiliary Surgery Hospital, \\ Second Military Medical University, Shanghai 200438; ${ }^{3}$ Department of General Surgery, No. 3 People's Hospital \\ Affiliated to Shanghai Jiaotong University School of Medicine (SJTU-SM), Shanghai 201900, P.R. China
}

Received January 16, 2014; Accepted March 17, 2014

DOI: $10.3892 /$ or.2014.3129

\begin{abstract}
Forkhead box M1 (FOXM1), a member of the Fox family of transcriptional factors, is considered to be an independent predictor of poor survival in many solid cancers. However, the underlying mechanism is not yet clear. The aim of the present study was to investigate the clinical significance of the correlation between FOXM1 and epithelial-mesenchymal transition (EMT) in non-small cell lung carcinoma and the possible mechanism responsible for FOXM1-induced EMT and metastasis. In the present study, expression levels of FOXM1 and EMT indicator proteins were determined by tissue microarray (TMA) and immunohistochemical staining, western blotting and reverse transcription-PCR (RT-PCR). Other cellular and molecular approaches including gene transfection, small interfering RNA (siRNA), and migration and invasion assays were utilized. Our results demonstrated that FOXM1 overexpression was statistically significantly associated with a higher TNM stage $(p=0.036)$, lymph node metastasis $(p=0.009)$ and a positive smoking history of the patients $(\mathrm{p}=0.044)$. Additionally, high expression of FOXM1 correlated with loss of E-cadherin expression $(p<0.001)$ and anomalous immunopositivity of Vimentin $(\mathrm{p}=0.002)$. Moreover, patient survival analysis demonstrated that high expression of FOXM1 $(\mathrm{p}=0.043)$ and the presence of lymph node metastasis $(\mathrm{p}=0.042)$ were independent prognostic factors for non-small cell lung cancer (NSCLC). Furthermore, various in vitro experiments indicated that overexpression or knockdown of FOXM1 expression altered EMT through activation or inhibition of the $\mathrm{AKT} / \mathrm{p} 70^{\mathrm{S} 6 \mathrm{~K}}$ signaling pathway.
\end{abstract}

Correspondence to: Dr Bin Jiang, Department of Oncology, No. 3 People's Hospital Affiliated to Shanghai Jiaotong University School of Medicine (SJTU-SM), Shanghai 201900, P.R. China

E-mail: dr_jiang@yeah.net

${ }^{*}$ Contributed equally

Key words: FOXM1, epithelial-mesenchymal transition, E-cadherin, Vimentin, non-small cell lung cancer, AKT
Collectively, the results suggest that FOXM1 may be used as a prognostic indicator for patients with NSCLC and promotes metastasis by inducing EMT of lung cancer cells through activation of the AKT/p $70^{\mathrm{S} 6 \mathrm{~K}}$ pathway. Therefore, we suggest that FOXM1 may be a potential target for lung cancer therapy.

\section{Introduction}

Non-small cell lung cancer (NSCLC) is the leading cause of cancer-related mortality worldwide and is associated with a high rate of metastasis (1). Although metastasis is responsible for as much as $90 \%$ of cancer-associated deaths, it remains the most poorly understood aspect of cancer pathogenesis (2).

Forkhead box M1 (FOXM1), a member of the Fox family of transcriptional factors, belongs to a group of evolutionarily conserved transcriptional regulators that are characterized by the presence of a DNA-binding domain called the Forkhead box or the winged helix domain $(3,4)$. Generally speaking, FOXM1 plays a critical role in cell cycle progression by regulating $\mathrm{G} 1 / \mathrm{S}$ and $\mathrm{G} 2 / \mathrm{M}$ phase transitions of the cell cycle and ensuring the proper execution of mitotic cell division (3-5). It has been reported to be overexpressed in a variety of tumors, including lung, liver, breast and bladder cancer, clear cell renal cell carcinoma and early stage cervical cancer (6-11). Furthermore, increased expression of FOXM1 has been associated with poor prognosis in cancer patients and is considered to be an independent predictor of poor survival in many solid cancers (6,7,10-14). Importantly, downregulation of FOXM1 leads to the inhibition of invasion of pancreatic cancer (15), prostate cancer (16), clear cell renal cell carcinoma (14) and NSCLC (12) cells.

Epithelial-mesenchymal transition (EMT) is an essential phenotypic event during embryonic development, tissue remodeling and wound healing, and plays an essential role in tumor invasion and metastasis (17-19). EMT is also a reversible process that often occurs at the invasive front of many metastatic cancers (20). Loss of E-cadherin and relocalization of $\beta$-catenin from the membrane to the nucleus are frequently observed in tumor cells undergoing EMT $(17,21)$. Additionally, expression of the intermediate filament protein Vimentin, which is known to induce changes in cell motility, is a classic 
marker of EMT (22). Although both FOXM1 and EMT play important roles in the metastasis of cancer, the interaction between them remains unclear.

The PI3K/AKT pathway regulates EMT, which is a fundamental event that is thought to predict tissue invasion and metastatic potential $(23,24)$. PI3K and AKT also promote tissue invasiveness and downregulate expression of E-cadherin, a key marker of EMT (25-28). Additionally, Park et al found that FOXM1b induced an EMT-like phenotype by activating the AKT-Snail pathway in hepatocellular carcinoma (HCC) (29). However, there is no report on the important role of AKT in FOXM1-induced EMT in NSCLC.

In the present study, we sought to determine whether FOXM1 expression is associated with EMT in NSCLC specimens and whether these factors can predict the clinical outcome and survival of these cancer patients. In addition, we examined whether a strong correlation exists between FOXM1 expression and the EMT process in NSCLC cells and whether the AKT signaling pathway is involved in FOXM1-regulated EMT.

\section{Materials and methods}

Tissue samples and tissue microarray construction. The preparation of tissue specimens and the construction of the tissue microarray (TMA) were performed as described previously (30).

Immunohistochemistry. Immunohistochemical analysis of TMA slides was carried out as previously reported (30). The following primary monoclonal antibodies were used: FOXM1 rabbit mAb (1:100; Santa Cruz Biotechnology, Santa Cruz, CA, USA), E-cadherin and Vimentin rabbit mAb (1:100; Cell Signaling Technology, Beverly, MA, USA). The intensity score was defined as follows: no signal, 0 ; weak, 1; moderate, 2 and intense, 3 . The score was based on the fraction of immunoreactive cells $(0-100 \%)$. The total score was calculated by multiplying the intensity score and the fraction score, yielding a total score ranging from 0 to $300 \%$. For statistical analyses, scores of $0-100 \%$ were considered low expression, and scores of $101 \%-300 \%$ were considered high expression. Two of the pathologists, without prior knowledge of the clinical data, independently graded the staining intensity in all cases.

Cell culture, reagents and treatments. The lung cancer cell lines A549 and H1299 were cultured in Dulbecco's modified Eagle's minimum (DMEM) and H1650, H1975 in RPMI-1640 medium (both from Gibco-BRL), respectively, supplemented with $10 \%$ fetal bovine serum (FBS) (HyClone Inc., USA). These cell lines were incubated under standard conditions at $37^{\circ} \mathrm{C}$ in a humidified atmosphere containing $5 \% \mathrm{CO}_{2}$. The PI3K inhibitor LY294002 $(10 \mu \mathrm{mol} / \mathrm{l})$ and the activator epidermal growth factor (EGF) $(0.1 \mathrm{ng} / \mathrm{ml})$ were obtained from Sigma-Aldrich (St. Louis, MO, USA).

Western blotting and antibodies. Equal amounts of protein (50-200 $\mu \mathrm{g} /$ lane) were extracted from whole-cell lysates and were subjected to sodium dodecyl sulfate-polyacrylamide gel electrophoresis (SDS-PAGE). After being transferred to polyvinylidene difluoride (PVDF) membranes (Millipore, USA), the proteins were incubated with primary antibodies [FOXM1 rabbit mAb (1:500; Santa Cruz Biotechnology), E-cadherin, Vimentin, AKT, p-AKT (Ser473), p70 ${ }^{\mathrm{S} 6 \mathrm{~K}}$ or p-p70 ${ }^{\mathrm{S6K}}$ (Thr389) rabbit mAb (1:1,000; Cell Signaling Technology)], followed by appropriate HRP-conjugated secondary antibodies. Immunoreactive proteins were detected using the enhanced chemiluminescence detection kit (Thermo Scientific, Rockford, IL, USA).

$R T-P C R$. Total RNA of the cultured cells was isolated with TRIzol reagent according to the manufacturer's protocol (Invitrogen, Carlsbad, CA, USA) and was then resuspended in diethylpyrocarbonate (DEPC)-treated water. Reverse transcription was performed according to the manufacturer's instructions (Takara, Dalian, China). The primer sequences utilized were: FOXM1 sense, 5'-AGCGACAGGTTAAGGTTGAG-3' and antisense, 5'-GTGCTGTTGATGGCGAATTG-3'; E-cadherin sense, 5'-TGCCCAGAAAATGAAAAAGG-3' and antisense, 5'-GTGTATGTGGCAATGCGTTC-3'; Vimentin sense, 5'-GA GAACTTTGCCGTTGAAGC-3' and antisense, 5'-CTCAAT GTCAAGGGCCATCT-3'; GAPDH sense, 5'-ACGGATTTG GTCGTATTGGGCG-3' and antisense, 5'-CTCCTGGAA GATGGTGATGG-3'. The PCR products were separated by $1.5 \%$ agarose gel electrophoresis.

Transwell migration and invasion assays. In vitro, migration assays were performed in a Transwell chamber (Corning Costar, USA) containing a membrane filter $(8-\mu \mathrm{m}$ pore size). To measure cell invasion overnight, Transwell filters were coated on the lower chamber with $5 \mu \mathrm{g} / \mathrm{ml}$ of BioCoat Matrigel (BD Biosciences, USA) to reconstitute the matrix of the basement membrane. Cells were seeded at a density of $2.0 \times 10^{4} /$ insert in $200 \mu \mathrm{l}$ serum-free medium and transferred to wells filled with $600 \mu \mathrm{l}$ culture medium containing $10 \%$ FBS as a chemoattractant. After $24 \mathrm{~h}$ of incubation, noninvading cells on the top of the membrane were removed by scraping. Invading cells on the bottom of the membrane were fixed in $4 \%$ paraformaldehyde and stained with $0.05 \%$ crystal violet. The number of invading cells was then counted in nine random high power fields/well at x200 magnification under a light microscope.

Transient transfection of FOXM1 siRNA. FOXM1-specific small interfering RNA (siRNA) oligonucleotides were purchased from GenePharma (Shanghai GenePharma Co., Shanghai). The sequences of the double-stranded siRNA oligonucleotides utilized were: 5'GGACCACUUUCCCUA CUUUTT3' (sense) and 5'AAAGUAGGGAAAGUGGUC CTT3' (antisense). The negative control siRNA sequences were: 5'UUCUCCGAACGUGUCACGUTT3' (sense) and 5'ACGUGACACGUUCGGAGAATT3' (antisense). Human lung carcinoma H1975 and H1299 cells which were tested to have high FOXM1 expression were transfected with siRNA (100 nM) using the DharmaFECT4 siRNA transfection reagent (Dharmacon, Chicago, IL, USA) according to the manufacturer's protocol.

Stable transfection of FOXM1. Plasmid (EX-Z5438-LV135) and Lenti-Pac HIV Expression Packaging kit were purchased from GeneCopoeia Inc. (Guangzhou, China). Human lung carcinoma H1650 and A549 cells which were tested to have 
Table I. Correlation between expression of FOXM1 and EMT indicator proteins and clinicopathological factors of the NSCLC patients.

\begin{tabular}{|c|c|c|c|c|}
\hline Characteristics & Total (\%) & $\begin{array}{l}\text { High FOXM1 } \\
\text { expression, n (\%) }\end{array}$ & $\begin{array}{l}\text { High E-cadherin } \\
\text { expression, } \mathrm{n}(\%)\end{array}$ & $\begin{array}{l}\text { High Vimentin } \\
\text { expression, } \mathrm{n}(\%)\end{array}$ \\
\hline \multicolumn{5}{|l|}{ Gender } \\
\hline Female & $30(44.1)$ & $18(60.0)$ & $15(50.0)$ & $10(33.3)$ \\
\hline Male & $38(55.9)$ & $25(65.8)$ & $12(31.6)$ & $7(18.4)$ \\
\hline \multicolumn{5}{|l|}{ Age (years) } \\
\hline Mean & 59.44 & & & \\
\hline$<60$ & $29(42.6)$ & $17(58.6)$ & $14(48.3)$ & $5(17.2)$ \\
\hline$\geq 60$ & $39(57.4)$ & $26(66.7)$ & $13(33.3)$ & $12(30.8)$ \\
\hline \multicolumn{5}{|l|}{ Smoking status } \\
\hline No & $30(44.1)$ & $15(50.0)$ & $14(46.7)$ & $5(16.7)$ \\
\hline Yes & $38(55.9)$ & $28(73.7)^{\mathrm{a}}$ & $13(34.2)$ & $12(31.6)$ \\
\hline \multicolumn{5}{|l|}{ Histologic characterisation } \\
\hline Adenocarcinoma & $33(48.5)$ & $20(60.6)$ & $14(42.4)$ & $6(18.2)$ \\
\hline Squamous carcinoma & $21(30.9)$ & $12(57.1)$ & $9(42.9)$ & $3(14.2)$ \\
\hline Adenosquamous carcinoma & $14(20.6)$ & $11(78.6)$ & $4(28.6)$ & $8(57.1)^{\mathrm{b}}$ \\
\hline \multicolumn{5}{|l|}{ Differentiation } \\
\hline High/moderate & $39(57.3)$ & $21(53.8)$ & $17(43.6)$ & $10(25.6)$ \\
\hline Low & $29(42.7)$ & $22(75.9)$ & $10(34.5)$ & $7(24.1)$ \\
\hline \multicolumn{5}{|l|}{ TNM stage } \\
\hline $\mathrm{I}+\mathrm{II}$ & $27(39.7)$ & $13(48.1)$ & $15(55.6)$ & $2(7.4)$ \\
\hline $\mathrm{III}+\mathrm{IV}$ & $41(60.3)$ & $30(73.2)^{\mathrm{a}}$ & $12(29.3)^{\mathrm{a}}$ & $15(36.6)^{\mathrm{a}}$ \\
\hline \multicolumn{5}{|l|}{ Lymph node metastasis } \\
\hline Negative & $27(39.7)$ & $12(44.4)$ & $16(59.3)$ & $2(7.4)$ \\
\hline Positive & $41(60.3)$ & $31(75.6)^{\mathrm{a}}$ & $11(26.8)^{\mathrm{a}}$ & $15(36.6)^{\mathrm{a}}$ \\
\hline
\end{tabular}

${ }^{\mathrm{a} p}$-value $<0.05$. ${ }^{\mathrm{b}} \mathrm{p}$-value $<0.05$ vs. adenocarcinoma or squamous carcinoma. FOXM1, Forkhead box M1; EMT, epithelial-mesenchymal transition; NSCLC, non-small cell lung cancer.

low FOXM1 expression were stably transfected according to the manufacturer's instructions. The presence of stable transfectants was confirmed by RT-PCR and western blotting.

Statistical analyses. A comparison of clinicopathologic characteristics of the patients was evaluated using the Chi-square test. Overall survival was defined as the length of time from the date of diagnosis to the date of death or the date at which patients were last known to be alive. Survival analysis data were collected until the 1st of January 2012, and the Kaplan-Meier method was used to calculate overall survival. The differences between the survival curves were analyzed using the log-rank test. Spearman rank correlation coefficients were used to quantify the correlations between the expression of FOXM1 and EMT indicator proteins. Univariate analysis was performed using the log-rank test. Multivariate analysis was performed using the Cox proportional hazards model. All factors with a value of $\mathrm{p}<0.05$ by univariate analysis were included in the multivariate analysis. In the analyses, $\mathrm{p}<0.05$ was considered to indicate a statistically significant result. Statistical analyses were performed using SPSS 17.0 software (SPSS, Inc., Chicago, IL, USA).

\section{Results}

Increased expression of FOXM1 is associated with EMT in NSCLC tissues. The clinicopathological characteristics of the patients were previously reported in detail (30) and are summarized in Table I. The overall duration of the follow-up of these patients ranged from 1 to 60 months.

We sought to investigate the expression levels of the FOXM1 and EMT indicator proteins in NSCLC specimens and correlate these results with the patient clinical and pathologic findings. Immunohistochemical (IHC) analyses of tissue array slides were used to determine the expression levels of FOXM1, E-cadherin and Vimentin proteins. Either nuclear expression or mixed nuclear and cytoplasmic expression of FOXM1 in tumor cells was defined as positive immunoreactivity for FOXM1. Positive immunostaining for E-cadherin was localized to the cell membrane, whereas Vimentin was observed in the cytoplasm of cancer cells. Representative patterns of IHC staining are illustrated in Fig. 1.

As shown in Table I, high levels of expression of FOXM1, E-cadherin and Vimentin were observed in 43/68 (63.2\%), $27 / 68(39.7 \%)$ and $17 / 68(25.0 \%)$ specimens of NSCLC, 
A
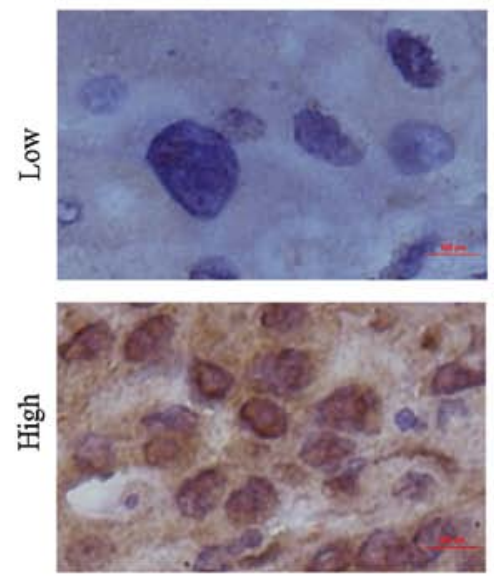

E-cadherin
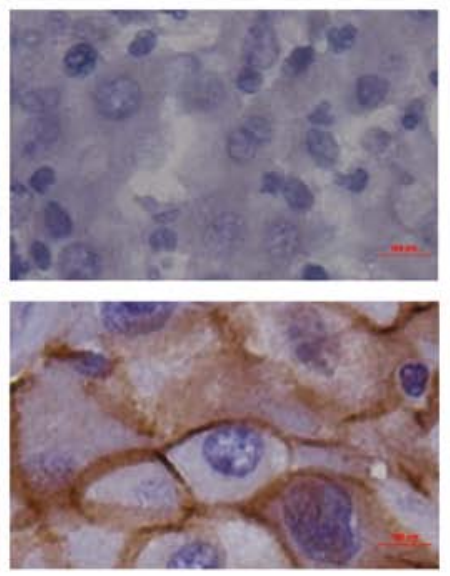

Vimentin
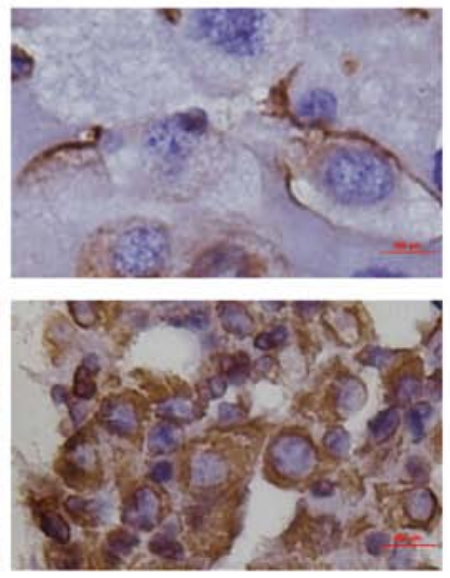

B

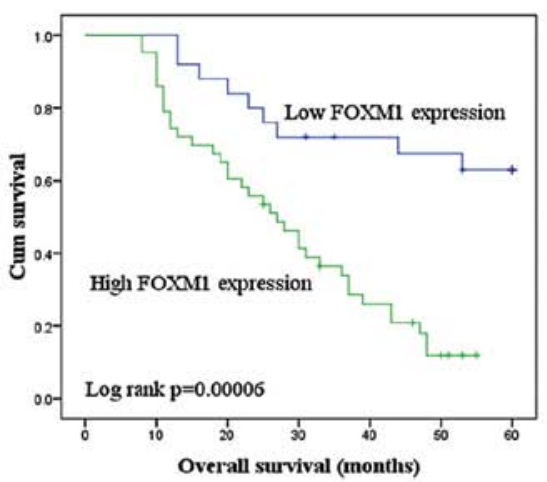

C

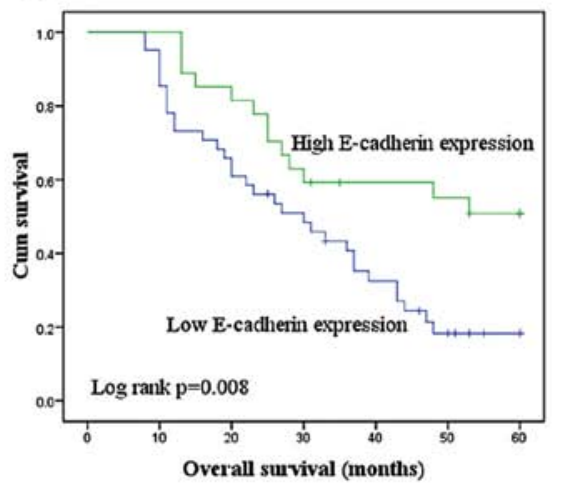

D

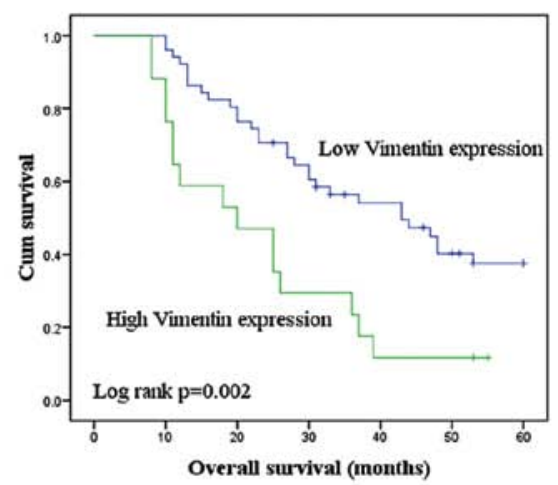

Figure 1. Representative IHC images showing expression of FOXM1 and EMT-related biomarkers in TMA sections of NSCLC and corresponding Kaplan-Meier survival curves. (A) The image shows representative NSCLC tissues exhibiting low and high levels of expression for FOXM1, E-cadherin and Vimentin. Scale bar, $100 \mu \mathrm{m}$. (B-D) Kaplan-Meier survival analyses of overall survival of NSCLC patients with respect to their level of expression of FOXM1, E-cadherin and Vimentin (B, FOXM1; C, E-cadherin and D, Vimentin). FOXM1, Forkhead box M1; EMT, epithelial-mesenchymal transition; TMA, tissue microarray; NSCLC, non-small cell lung cancer.

respectively. Notably, statistically significant correlations were demonstrated between FOXM1 and the smoking history of the patients $(\mathrm{p}=0.044)$, TNM stage $(\mathrm{p}=0.036)$ and lymph node metastasis status $(\mathrm{p}=0.009)$. Tumors from patients with a positive smoking history, advanced TNM stage and lymph node metastasis were characterized by higher FOXM1 expression compared with those without these features. Expression levels of E-cadherin and Vimentin were significantly associated with advanced TNM stage and lymph node metastasis. In addition, Vimentin expression was found to be significantly greater in lung adenosquamous carcinoma than that in squamous cell carcinoma.

Increased expression of FOXM1 is correlated with EMT indicator proteins E-cadherin and Vimentin in NSCLC tissues. To investigate the association between the expression of FOXM1 and EMT indicator proteins in NSCLC specimens, Spearman correlation analysis was used. As shown in Table II, a high expression level of FOXM1 was found to correlate with a loss of E-cadherin expression $(r=-0.69, p<0.001)$ and anomalous immunoreactivity of Vimentin $(r=0.370, p=0.002)$ in the NSCLC samples. Therefore, FOXM1 expression was significantly correlated with EMT in the NSCLC tissues.
Table II. Spearman correlation analysis between expression of FOXM1 and EMT indicator proteins in NSCLC.

\begin{tabular}{lrrrr}
\hline & \multicolumn{2}{c}{$\begin{array}{c}\text { FOXM1 } \\
\text { expression }\end{array}$} & & \\
\cline { 2 - 3 } EMT indicator proteins & Low & High & P-value & R-value \\
\hline E-cadherin expression & & & & \\
$\quad$ Low & 4 & 37 & $<0.001$ & -0.690 \\
High & 21 & 6 & & \\
Vimentin expression & & & & \\
$\quad$ Low & 24 & 27 & 0.002 & 0.370 \\
High & 1 & 16 & & \\
\hline
\end{tabular}

FOXM1, Forkhead box M1; EMT, epithelial-mesenchymal transition; NSCLC, non-small cell lung cancer.

The expression levels of FOXM1, E-cadherin and Vimentin predict overall survival time of NSCLC patients. Overall survival analysis was used to determine the existence of a 
Table III. Univariate and multivariate analyses of overall survival.

\begin{tabular}{|c|c|c|c|c|c|c|c|}
\hline \multirow[b]{2}{*}{ Characteristics } & \multicolumn{3}{|c|}{ Univariate analysis } & \multirow{2}{*}{$\begin{array}{l}\text { Median survival } \\
\quad \text { (months) }\end{array}$} & \multicolumn{3}{|c|}{ Multivariate analysis } \\
\hline & HR & $95 \% \mathrm{CI}$ & P-value & & HR & $95 \%$ CI & P-value \\
\hline Gender & 0.897 & $0.496-1.622$ & 0.716 & & - & - & - \\
\hline Female & & & & 36.733 & & & \\
\hline Male & & & & 35.531 & & & \\
\hline Age (years) & 1.811 & $0.980-3.348$ & 0.058 & & - & - & - \\
\hline$<60$ & & & & 42.151 & & & \\
\hline$\geq 60$ & & & & 31.498 & & & \\
\hline Smoking status & 1.152 & $0.639-2.076$ & 0.637 & & - & - & - \\
\hline No & & & & 37.5 & & & \\
\hline Yes & & & & 34.93 & & & \\
\hline Histologic characterisation & 0.900 & $0.613-1.322$ & 0.593 & & - & - & - \\
\hline Adenocarcinoma & & & & 33.391 & & & \\
\hline Squamous carcinoma & & & & 40.899 & & & \\
\hline Adenosquamous carcinoma & & & & 35.714 & & & \\
\hline Differentiation & 0.540 & $0.300-0.972$ & 0.040 & & 0.606 & $0.320-1.148$ & 0.124 \\
\hline High or moderate & & & & 40.812 & & & \\
\hline Low & & & & 29.610 & & & \\
\hline TNM stage & 2.996 & $1.532-5.858$ & 0.001 & & 1.247 & $0.495-3.143$ & 0.639 \\
\hline $\mathrm{I}+\mathrm{II}$ & & & & 45.591 & & & \\
\hline $\mathrm{III}+\mathrm{IV}$ & & & & 29.733 & & & \\
\hline Lymph node metastasis & 3.395 & $1.732-6.654$ & 0.0001 & & 2.595 & $1.034-6.510$ & 0.042 \\
\hline Negative & & & & 47.113 & & & \\
\hline Positive & & & & 28.548 & & & \\
\hline FOXM1 expression & 4.099 & $1.934-8.688$ & 0.00023 & & 2.950 & $1.036-8.397$ & 0.043 \\
\hline Low & & & & 47.645 & & & \\
\hline High & & & & 28.610 & & & \\
\hline E-cadherin expression & 0.429 & $0.223-0.824$ & 0.001 & & 1.181 & $0.500-2.789$ & 0.704 \\
\hline Low & & & & 31.297 & & & \\
\hline High & & & & 43.344 & & & \\
\hline Vimentin expression & 2.596 & $1.386-4.864$ & 0.003 & & 1.287 & $0.642-2.580$ & 0.477 \\
\hline Low & & & & 40.012 & & & \\
\hline High & & & & 23.882 & & & \\
\hline
\end{tabular}

HR, hazard ratio; CI, confidence interval; FOXM1, Forkhead box M1.

correlation between the expression of FOXM1 and EMT indicator proteins and patient prognosis. Kaplan-Meier survival curves for overall survival with respect to FOXM1 and EMT indicator protein expression are shown in Fig. 1. These plots demonstrated that NSCLC patients expressing low levels of FOXM1 and Vimentin but high levels of E-cadherin survived significantly longer than patients with NSCLC expressing high FOXM1 and Vimentin but low E-cadherin levels.

In addition, a Cox proportional hazards model was applied to estimate the effect of FOXM1 and EMT indicator proteins on patient survival. Univariate Cox regression analysis identified tumor differentiation, TNM stage, lymph node metastasis, and expression of FOXM1, E-cadherin and Vimentin as significant prognostic factors (Table III). The crude hazard ratio
(HR) of NSCLC with high FOXM1 expression compared with low FOXM1 expression was 4.099 (95\% confidence interval 1.934-8.688, $\mathrm{p}=0.00006)$. In other words, high FOXM1 expression in NSCLC patients increased the likelihood of lung cancer-related death 4-fold above that of a low FOXM1 status. Using multivariable analysis, lymph node metastasis and FOXM1 were found to be statistically significant independent prognostic factors for overall survival $(\mathrm{p}=0.042$ and $\mathrm{p}=0.043$, respectively). These findings suggest that FOXM1 expression behaves as an independent and statistically significant predictor for poor patient survival.

Stable overexpression of FOXM1 protein induces EMT in NSCLC cells. Our data provide clinical evidence supporting 
A
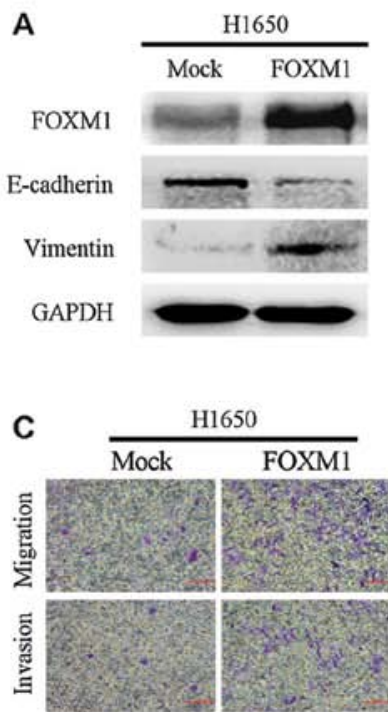
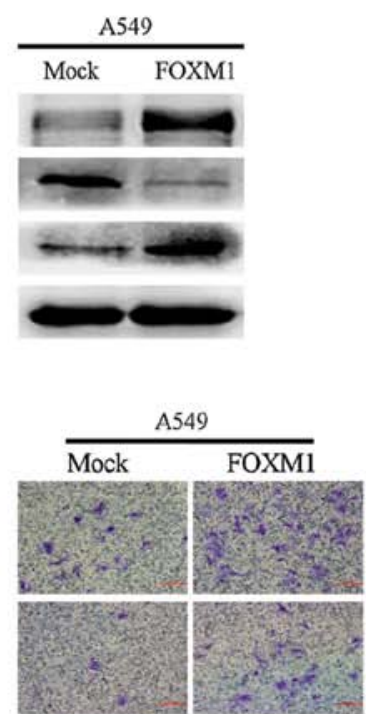
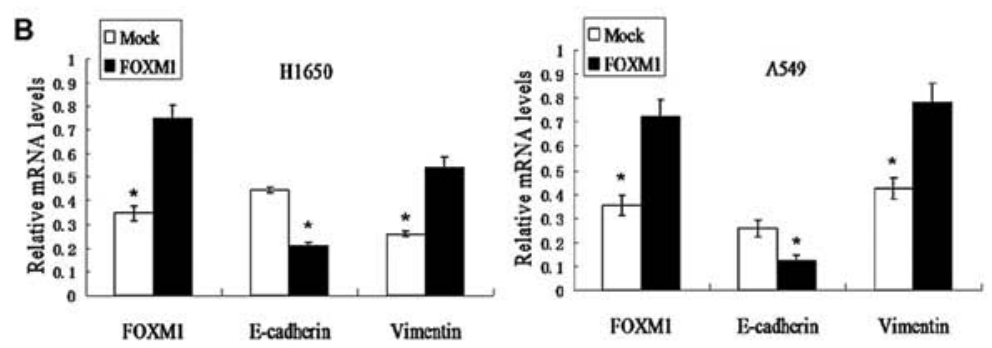
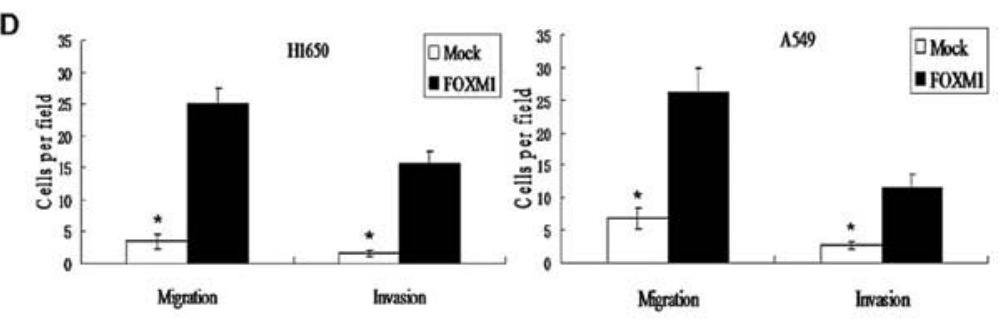

Figure 2. Effects of lentiviral-mediated FOXM1 overexpression on EMT and the invasive capacity of NSCLC cells. (A) H1650 and A549 cells transfected with a control or FOXM1-specific lentivirus were harvested, lysed and then subjected to SDS-PAGE, transferred to membranes, and blotted with the indicated antibody with GAPDH as a loading control. (B) Quantification of the target mRNA bands relative to GAPDH is shown in the histograms. (C) Effects of FOXM1 overexpression on the invasive capacity of $\mathrm{H} 1650$ and A549 cells by Transwell assay. The invasive cells were stained and counted under a microscope. Scale bar, $500 \mu \mathrm{m}$. (D) Quantitative results for the transmembrane ability of each group of cells. "p<0.05. FOXM1, Forkhead box M1; EMT, epithelial-mesenchymal transition; NSCLC, non-small cell lung cancer.
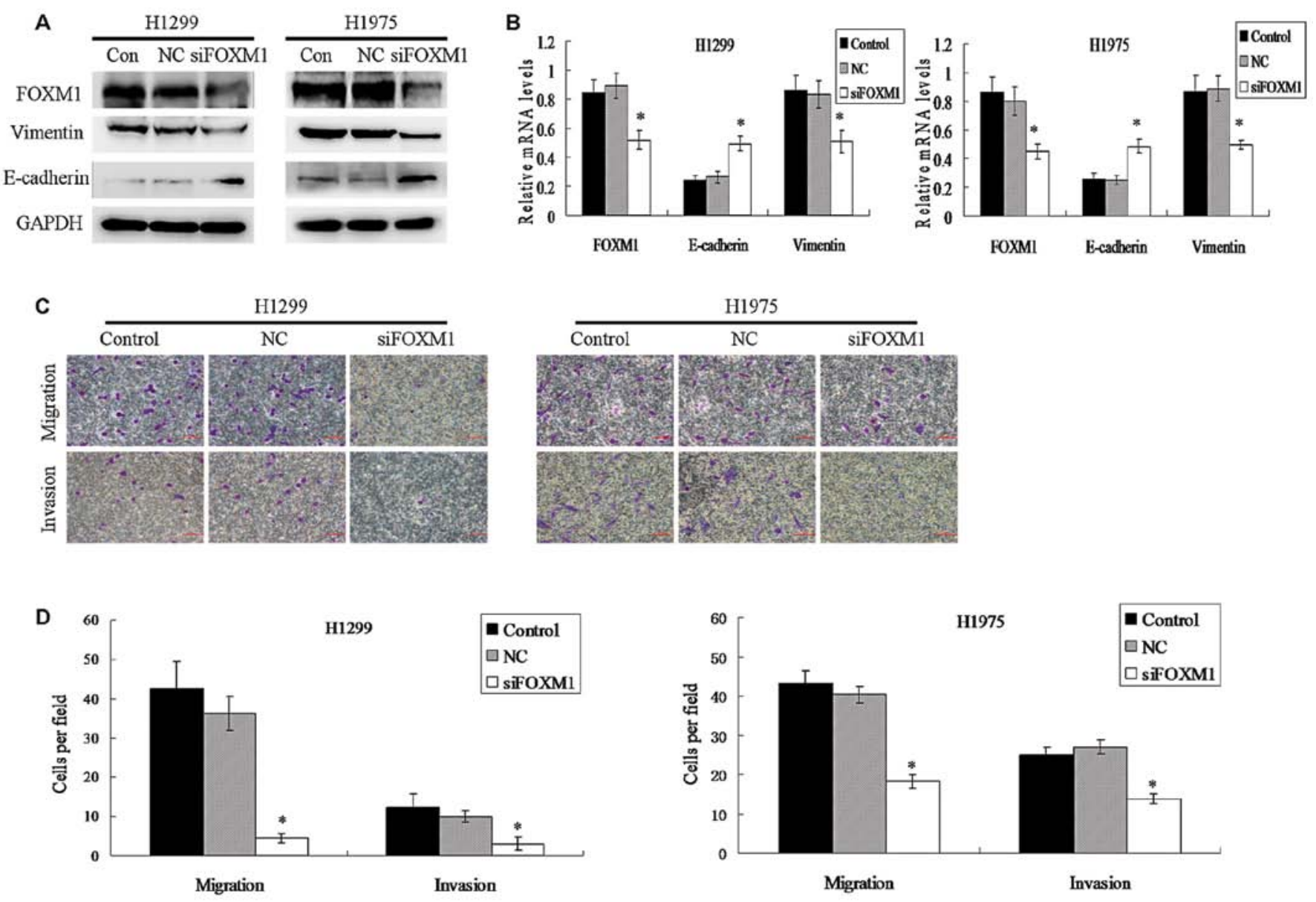

Figure 3. Effects of siRNA-mediated FOXM1 inhibition on EMT and the invasive capacity of NSCLC cells. (A) One pair of siRNA specifically against FOXM1 or negative control siRNA (NC) was transiently transfected into H1299 or H1975 cells. Two days after transfection, FOXM1 and EMT indicator proteins were detected by western blotting with GAPDH used as a loading control. (B) Quantification of the target mRNA bands relative to GAPDH is shown in the histograms. (C) Effects of siRNA-mediated FOXM1 inhibition on the invasive capacity of H1299 and H1975 cells by Transwell assay. The invasive cells were stained and counted under a microscope. Scale bar, $500 \mu \mathrm{m}$. (D) Quantitative results for the transmembrane ability of each group of cells, ${ }^{*} \mathrm{p}<0.05$. FOXM1, Forkhead box M1; EMT, epithelial-mesenchymal transition; NSCLC, non-small cell lung cancer; siRNA, small interfering RNA. 
$\mathbf{A}$

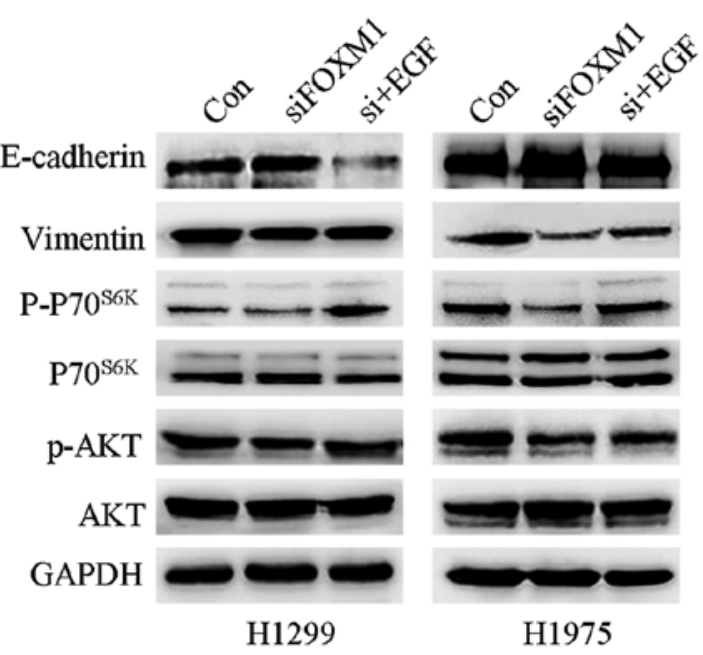

C

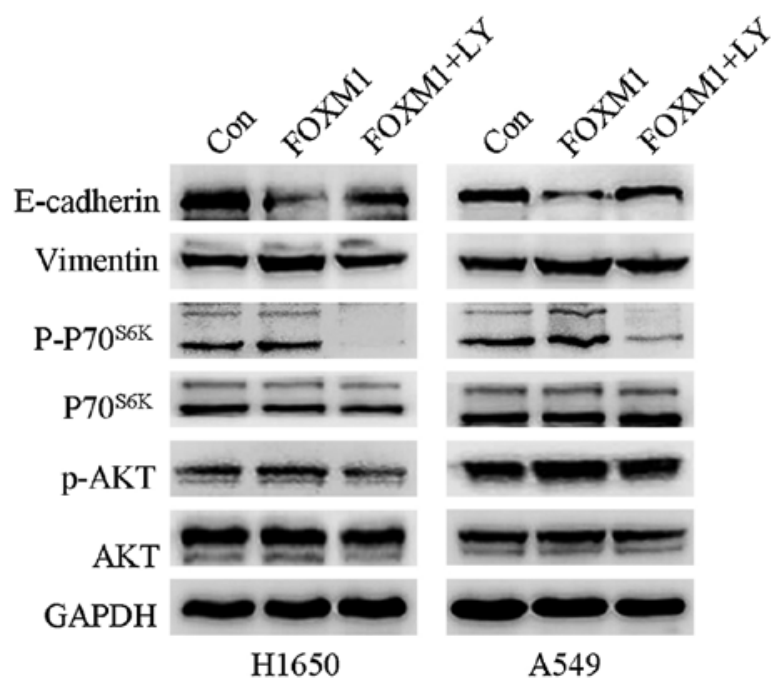

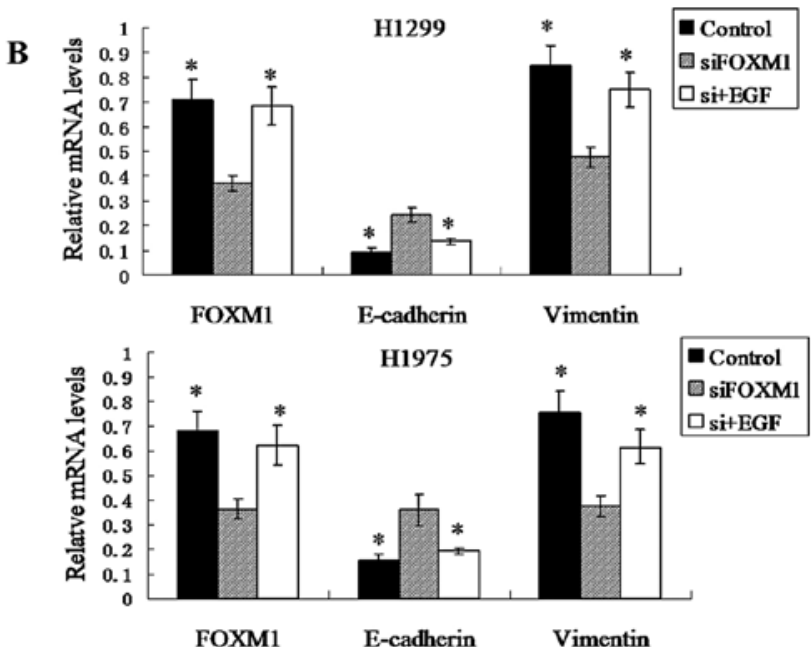

D

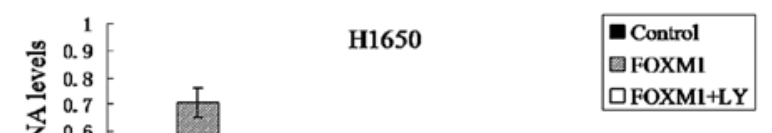

\section{密}
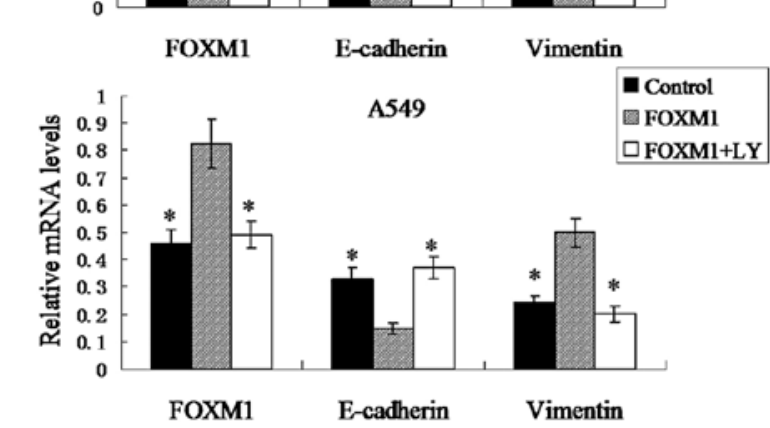

Figure 4. Effects of inhibition and activation of the AKT/p70 ${ }^{\mathrm{s} 6 \mathrm{~K}}$ pathway on EMT of NSCLC cells. H1299 and H1975 cells transiently transfected with siRNA specifically against FOXM1 were treated with EGF $(0.1 \mathrm{ng} / \mathrm{ml})$ for $48 \mathrm{~h}$. Effect of the kinase activator on the expression of EMT indicator proteins was detected by (A) western blotting and (B) RT-PCR, ${ }^{*}$ p<0.05 vs. siFOXM1 or FOXM1. H1650 and A549 cells transfected with the FOXM1-specific lentivirus were treated with LY294002 (LY) $(10 \mu \mathrm{mol} / \mathrm{l})$ for $48 \mathrm{~h}$. Effect of the kinase inhibitor on the expression of EMT indicator proteins was detected by (C) western blotting and (D) RT-PCR. EMT, epithelial-mesenchymal transition; NSCLC, non-small cell lung cancer; siRNA, small interfering RNA; EGF, epidermal growth factor.

the hypothesis that high FOXM1 expression is associated with EMT and poor survival of patients with NSCLC. Furthermore, FOXM1 has been implicated in the regulation of the EMT process in pancreatic cancer cells (31). To test whether FOXM1 expression induces EMT in NSCLC cells, human lung carcinoma $\mathrm{H} 1650$ and A549 cells were stably transfected with a lentiviral vector expressing FOXM1. Following transfection, protein and mRNA levels of E-cadherin were found to be decreased while those of Vimentin were increased in these cells (Fig. 2A and B). To further characterize the EMT process in FOXM1-expressing cells, the migration capacity of FOXM1-transfected cells and control lentivirus-transfected (mock) cells were compared using Transwell chambers. The elevated expression of FOXM1 promoted the migration and invasion of transfected cells when compared with the mock cells (Fig. 2C and D). These data suggest that FOXM1 may promote metastasis by inducing the EMT process in NSCLC cell lines.
Knockdown of FOXM1 expression inhibits the EMT process of NSCLC cells. To test whether FOXM1 expression was necessary for the EMT process of NSCLC cells, the human NSCLC cell lines H1299 and H1975 were transiently transfected with negitive control siRNA or FOXM1-specific siRNA to suppress the expression of FOXM1. Knockdown of FOXM1 gene expression increased the expression of E-cadherin while decreasing the expression of Vimentin both at the protein and mRNA levels in the transfected cells (Fig. 3A and B). As shown in Fig. 3C and D, knockdown of FOXM1 markedly reduced the migration and invasion of the transfected cells. These results suggest that FOXM1 expression is critical for the EMT process in lung cancer cells.

Overexpression and knockdown of FOXM1 expression regulate EMT through activation and inhibition of the $A K T / p 70^{S 6 K}$ signaling pathway. To explore the mechanism whereby FOXM1 expression enhances EMT, we examined the effects 
of FOXM1 on the expression and phosphorylation of AKT and $\mathrm{p} 70^{\mathrm{S} 6 \mathrm{~K}}$. We found that knockdown of endogenous FOXM1 inhibited the phosphorylation levels of AKT and $\mathrm{p} 70^{\mathrm{s} 6 \mathrm{~K}}$ but not total AKT/ $\mathrm{p} 70^{\mathrm{S} 6 \mathrm{~K}}$. Conversely, overexpression of FOXM1 induced the opposite results.

To further investigate whether the activation of AKT pathway is responsible for the FOXM1-induced EMT in NSCLC, an inhibitor (LY294002) and an activator (EGF) of the PI3K/AKT pathway were used in experiments based on the observation that overexpression and knockdown of FOXM1 can activate and inhibit this pathway, respectively. As shown in Fig. 4, FOXM1-expressing cells treated with the inhibitor LY294002 showed reduced expression of Vimentin but increased E-cadherin expression. In contrast, EMT was promoted in siRNA-transfected cells incubated with EGF for $48 \mathrm{~h}$. These results suggest that activation of the AKT/p70 pathway plays an important role in FOXM1-induced EMT in NSCLC.

\section{Discussion}

In the present study, we demonstrated that high levels of expression of FOXM1 and EMT indicator proteins are significantly associated with several clinicopathologic variables. We also demonstrated, for the first time, the existence of a significant association between FOXM1 and EMT indicator protein expression. Additionally, the present study demonstrated that FOXM1 expression may be an independent prognostic indicator for overall survival of patients with NSCLC. Furthermore, our results suggest that FOXM1 expression may promote NSCLC metastasis by inducing EMT of tumors cells via the activation of the $\mathrm{AKT} / \mathrm{p} 70^{\mathrm{s} 6 \mathrm{~K}}$ pathway in lung cancer cells.

As previously mentioned, the existence of a significant association between FOXM1 expression and poor prognosis has been reported in many human types of cancers, including lung cancer $(6,12,32)$. In agreement with these reports, we found that FOXM1 is an independent prognostic indicator for overall survival of NSCLC patients. Our results also suggest that FOXM1 expression in tumor specimens is positively associated with TNM stage and the presence of lymph node metastasis. However, Liu et al (32) reported no association between FOXM1 expression and the clinical parameters studied, which raises questions about how tumor specimens were obtained and how IHC expression was analyzed.

There is considerable evidence that the presence of EMT signifies reduced survival of lung cancer patients (33-36). Previous studies reported that negative or low E-cadherin expression ranged from 46 to $75.21 \%$ in NSCLC specimens (37-40), which is similar to the low E-cadherin expression level of $60.3 \%$ found in the present study. Our results also showed that Vimentin expression was significantly associated with the presence of lymph node metastasis, TNM stage and the histologic type of the tumor. In contrast with our results, Dauphin et al (41) did not identify any association between Vimentin expression and lymph node metastasis. These differences may reflect the different study populations and varied clinical data quality.

To the best of our knowledge, this is the first report that high FOXM1 expression is significantly associated with EMT in NSCLC specimens. To extend the above results, we studied overexpression or knockdown of FOXM1 in lung cancer cell lines and found that FOXM1-mediated EMT results in the invasion and migration in vitro. Notably, Xu et al (12) reported that levels of E-cadherin and ZO-1 were reduced and that Vimentin and $\mathrm{N}$-cadherin expression levels were elevated in NSCLC cells expressing high levels of FOXM1. These results suggest that FOXM1 expression is associated with poor prognosis by regulating EMT in NSCLC.

Until recently, the precise molecular regulation of FOXM1 on EMT and metastasis was unclear. Previous research has shown that AKT has a distinct role in regulating EMT and cell migration (42). Furthermore, FOXM1 has been shown to activate the AKT-Snail pathway to promote cell migration and invasion in hepatocellular carcinoma (29) and early stage cervical cancer (11), while its role in NSCLC has not been reported. The present study demonstrated that FOXM1 activates the $\mathrm{AKT} / \mathrm{p} 70^{\mathrm{S} 6 \mathrm{~K}}$ signaling pathway thereby promoting EMT transition by enhancing cell migration and the invasive capacity in NSCLC. Furthermore, our research team is currently exploring the possible mechanisms involved in the regulation of PI3K/AKT by FOXM1.

In summary, our results suggest that FOXM1 expression is associated with a poor prognosis and EMT in NSCLC. In cell culture experiments, FOXM1 overexpression promoted migration and invasion by inducing the EMT of lung cancer cells via activation of the $\mathrm{AKT} / \mathrm{p} 70^{\mathrm{s} 6 \mathrm{~K}}$ pathway. The present study raises the possibility that FOXM1 may be a potential target for cancer therapy as it plays a crucial role in tumor migration and invasion.

\section{Acknowledgements}

This study was supported by the Shanghai Jiaotong University School of Medicine Science Fund Project (11XJ22014), the Hospital Foundation of No. 3 People's Hospital, which is affiliated with the Shanghai Jiaotong University School of Medicine (syz2011-05), the Education Fund for Outstanding Young Teachers of Shanghai (zzjdyx12111), and the Science and Technology Commission of Shanghai Municipality (10JC1409200).

\section{References}

1. Herbst RS, Heymach JV and Lippman SM: Lung Cancer. N Engl J Med 359: 1367-1380, 2008.

2. Chaffer CL and Weinberg RA: A perspective on cancer cell metastasis. Science 331: 1559-1564, 2011.

3. Laoukili J, Kooistra MR, Brás A, et al: FoxM1 is required for execution of the mitotic programme and chromosome stability. Nat Cell Biol 7: 126-136, 2005.

4. Laoukili J, Stahl M and Medema RH: FoxM1: at the crossroads of ageing and cancer. Biochim Biophys Acta 1775: 92-102, 2007.

5. Wierstra I and Alves J: FOXM1, a typical proliferation-associated transcription factor. Biol Chem 388: 1257-1274, 2007.

6. Yang DK, Son CH, Lee SK, Choi PJ, Lee KE and Roh MS: Forkhead box M1 expression in pulmonary squamous cell carcinoma: correlation with clinicopathologic features and its prognostic significance. Hum Pathol 40: 464-470, 2009.

7. Sun HC, Li M, Lu JL, et al: Overexpression of Forkhead box M1 protein associates with aggressive tumor features and poor prognosis of hepatocellular carcinoma. Oncol Rep 25: 1533-1539, 2011.

8. Bektas N, Haaf At, Veeck J, et al: Tight correlation between expression of the Forkhead transcription factor FOXM1 and HER2 in human breast cancer. BMC Cancer 8: 42, 2008. 
9. Liu D, Zhang Z and Kong CZ: High FOXM1 expression was associated with bladder carcinogenesis. Tumour Biol 34: $1131-1138,2013$

10. Wu XR, Chen YH, Liu DM, Sha JJ, Xuan HQ, Bo JJ and Huang YR: Increased expression of forkhead box M1 protein is associated with poor prognosis in clear cell renal cell carcinoma. Med Oncol 30: 346, 2013.

11. He SY, Shen HW, Xu L, et al: FOXM1 promotes tumor cell invasion and correlates with poor prognosis in early-stage cervical cancer. Gynecol Oncol 127: 601-610, 2012.

12. Xu N, Jia D, Chen W, et al: FoxM1 is associated with poor prognosis of non-small cell lung cancer patients through promoting tumor metastasis. PLoS One 8: e59412, 2013.

13. Chu XY, Zhu ZM, Chen LB, et al: FOXM1 expression correlates with tumor invasion and a poor prognosis of colorectal cancer. Acta Histochem 114: 755-762, 2012.

14. Xue YJ, Xiao RH, Long DZ, et al: Overexpression of FoxM1 is associated with tumor progression in patients with clear cell renal cell carcinoma. J Transl Med 10: 200, 2012.

15. Wang Z, Banerjee S, Kong D, Li Y and Sarkar FH: Downregulation of Forkhead Box M1 transcription factor leads to the inhibition of invasion and angiogenesis of pancreatic cancer cells. Cancer Res 67: 8293-8300, 2007.

16. Lynch TP, Ferrer CM, Jackson SR, Shahriari KS, Vosseller K and Reginato MJ: Critical role of $O$-linked $\beta$ - $N$-acetylglucosamine transferase in prostate cancer invasion, angiogenesis, and metastasis. J Biol Chem 287: 11070-11081, 2012.

17. Kalluri R and Weinberg RA: The basics of epithelial-mesenchymal transition. J Clin Invest 119: 1420-1428, 2009.

18. Thiery JP, Acloque H, Huang RY and Nieto MA: Epithelialmesenchymal transitions in development and disease. Cell 139: 871-890, 2009

19. Wu YD and Zhou BP: Snail: more than EMT. Cell Adh Migr 4: 199-203, 2010

20. Christofori G: New signals from the invasive front. Nature 441: 444-450, 2006.

21. Zeisberg $M$ and Neilson EG: Biomarkers for epithelial-mesenchymal transitions. J Clin Invest 119: 1429-1437, 2009.

22. Eriksson JE, Dechat T, Grin B, Helfand B, Mendez M, Pallari HM and Goldman RD: Introducing intermediate filaments: from discovery to disease. J Clin Invest 119: 1763-1771, 2009.

23. Cheng GZ, Park S, Shu S, et al: Advances of AKT pathway in human oncogenesis and as a target for anti-cancer drug discovery. Curr Cancer Drug Targets 8: 2-6, 2008.

24. Onoue T, Uchida D, Begum NM, Tomizuka Y, Yoshida H and Sato M: Epithelial-mesenchymal transition induced by the stromal cell-derived factor-1/CXCR4 system in oral squamous cell carcinoma cells. Int J Oncol 29: 1133-1138, 2006.

25. Grille SJ, Bellacosa A, Upson J, et al: The protein kinase Akt induces epithelial mesenchymal transition and promotes enhanced motility and invasiveness of squamous cell carcinoma lines. Cancer Res 63: 2172-2178, 2003.

26. Larue L and Bellacosa A: Epithelial-mesenchymal transition in development and cancer: role of phosphatidylinositol 3 kinase/AKT pathways. Oncogene 24: 7443-7454, 2005.

27. Schramek H, Feifel E, Marschitz I, Golochtchapova N, Gstraunthaler G and Montesano R: Loss of active MEK1-ERK1/2 restores epithelial phenotype and morphogenesis in transdifferentiated MDCK cells. Am J Physiol Cell Physiol 285: C652-C661, 2003
28. Thiery JP and Sleeman JP: Complex networks orchestrate epithelial-mesenchymal transitions. Nat Rev Mol Cell Biol 7: 131-142, 2006.

29. Park HJ, Gusarova G, Wang Z, et al: Deregulation of FoxM1b leads to tumour metastasis. EMBO Mol Med 3: 21-34, 2011.

30. Zhao M, Gao FH, Wang JY, Liu F, Yuan HH, Zhang WY and Jiang B: JAK2/STAT3 signaling pathway activation mediates tumor angiogenesis by upregulation of VEGF and bFGF in nonsmall-cell lung cancer. Lung Cancer 73: 366-374, 2011.

31. Bao B, Wang Z, Ali S, Kong D, et al: Over-expression of FoxM1 leads to epithelial-mesenchymal transition and cancer stem cell phenotype in pancreatic cancer cells. J Cell Biochem 112: 2296-2306, 2011.

32. Liu YQ, Guo RH, Liu LK, Gao W, Zhu CJ, Wei J and Shu YQ Correlation between expression of forkhead box M1 (FOXM1) and clinicopathological features and prognosis in patients with non-small cell lung cancer (NSCLC). Zhonghua Zhong Liu Za Zhi 33: 426-430, 2011 (In Chinese).

33. Li LP, Lu CH, Chen ZP, et al: Subcellular proteomics revealed the epithelial-mesenchymal transition phenotype in lung cancer. Proteomics 11: 429-439, 2011.

34. Wang G, Dong W, Shen H, Mu X, Li Z, Lin X, Liu Y and Du J: A comparison of Twist and E-cadherin protein expression in primary non-small-cell lung carcinoma and corresponding metastases. Eur J Cardiothorac Surg 39: 1028-1032, 2011.

35. Pirozzi G, Tirino V, Camerlingo R, et al: Epithelial to mesenchymal transition by TGF $\beta-1$ induction increases stemness characteristics in primary non small cell lung cancer cell line. PLoS One 6: e21548, 2011.

36. Soltermann A, Tischler V, Arbogast S, et al: Prognostic significance of epithelial-mesenchymal and mesenchymal-epithelial transition protein expression in non-small cell lung cancer. Clin Cancer Res 14: 7430-7437, 2008.

37. Zhao JQ, Sun FJ, Liu SS, et al: Expression of connexin 43 and E-cadherin protein and mRNA in non-small cell lung cancers in Chinese patients. Asian Pac J Cancer Prev 14: 639-643, 2013.

38. Zhang X, Wang Z, Kang Y, Li X, Ma X and Ma L: MCAM expression is associated with poor prognosis in non-small cell lung cancer. Clin Transl Oncol 16: 178-183, 2014.

39. Jin S, Zhu W, Shi Q, Zhang Z and Guo R: Clinicopathological significance of lymphatic vessel density marked by D2-40 and E-cadherin expression in non-small-cell lung cancer. Med Oncol 29: 3157-3161, 2012.

40. Ucvet A, Kul C, Gursoy S, Erbaycu AE, Kaya SO, Dinc ZA and Yucel N: Prognostic value of epithelial growth factor receptor, vascular endothelial growth factor, E-cadherin, and p120 catenin in resected non-small cell lung carcinoma. Arch Bronconeumol 47: 397-402, 2011 (In Spanish).

41. Dauphin M, Barbe C, Lemaire S, et al: Vimentin expression predicts the occurrence of metastases in non small cell lung carcinomas. Lung Cancer 81: 117-122, 2013.

42. Irie HY, Pearline RV, Grueneberg D, et al: Distinct roles of Akt1 and Akt2 in regulating cell migration and epithelial-mesenchymal transition. J Cell Biol 171: 1023-1034, 2005. 